\title{
Complications of Acute and Chronic Hyperglycemia
}

\author{
M Loredana Marcovecchio \\ University of Cambridge, Cambridge, UK
}

$\mathrm{H}$ yperglycemia is due to a dysregulation in the complex mechanisms implicated in glucose homeostasis. Chronic hyperglycemia, as measured by hemoglobin A1C (HDA1C), is a key risk factor for the development of microvascular and macrovascular complications, which in turn negatively influence the prognosis of patients with diabetes. Several studies have shown that acute hyperglycemia can add to the effect of chronic hyperglycemia in inducing tissue damage. Acute hyperglycemia can manifest as high fasting plasma glucose (FPG) or high postprandial plasma glucose (PPG) and can activate the same metabolic and hemodynamic pathways as chronic hyperglycemia. Glucose variability, as expressed by the intraday glucose fluctuations from peaks to nadirs, is another important parameter, which has emerged as an HDA1c-independent risk factor for the development of vascular complications, mainly in the context of type 2 diabetes. Treatments able to decrease HDA1c have been associated with positive effects in terms of reducing risk for the development and progression of complications. Further studies are required to clarify the impact of strategies more specifically targeting components of acute hyperglycemia, to improve outcomes in patients with diabetes.

\section{Keywords}

Hyperglycemia, complications,

vascular, acute, chronic

Disclosure: M Loredana Marcovecchio has nothing to disclose in relation to this article. No funding was received in the publication of this article. This study involves a review of the literature and did not involve any studies with human or animal subjects performed by any of the authors.

Authorship: Thel named author meets the International Committee of Medical Journal Editors (ICMJE) criteria for authorship of this manuscript, takes responsibility for the integrity of the work as a whole, and has given final approval to the version to be published.

open Access: This article is published under the Creative Commons Attribution Noncommercial License, which permits any non-commercial use, distribution, adaptation and reproduction provided the original author(s) and source are given appropriate credit.

Received: February 27, 2017

Accepted: March 30, 2017

Citation: US Endocrinology,

2017;13(1):Epub ahead of printx

Corresponding Author: M Loredana Marcovecchio, University of Cambridge, Box 116, Level 8, Cambridge Biomedical Campus, Hills Road, Cambridge, CB2 OQQ, UK. E:mIm45@medschl.cam.ac.uk

\section{From glucose homeostasis to hyperglycemia}

Glucose homeostasis is maintained by a complex neurohormonal system, which modulates peripheral glucose uptake, hepatic glucose production, and exogenous glucose utilization following food ingestion. ${ }^{12}$ This allows the maintenance of plasma glucose concentrations within normal range, with average values of around $90 \mathrm{mg} / \mathrm{dl}$ throughout a 24-hour period, postmeal concentration below $140 \mathrm{mg} / \mathrm{dl}$, and minimal values, such as those after moderate fasting or exercise, above $55 \mathrm{mg} / \mathrm{dd} .{ }^{1{ }^{12}}$

Hormones implicated in glucose regulation include insulin, glucagon, amylin, glucagon-like petide-1 (GLP-1), glucose-dependent insulinotropic peptide, epinephrine, cortisol, and growth hormone. ${ }^{3}$ These hormones act on several target tissues, including muscle, liver, adipocyte, and brain to regulate glucose levels. ${ }^{3}$

Insulin is a key glucoregulatory hormone, produced by pancreatic $\beta$-cells, whose levels are low during the fasting state, whereas they increase during the postprandial phase, when insulin stimulates utilization of dietary glucose by peripheral tissues, and in the meantime represses hepatic glucose production. ${ }^{4}$ Another important hormone regulating glucose metabolism is glucagon, produced by pancreatic $\alpha$-cells during fasting conditions, when it induces hepatic glucose production through the activation of glycogenolysis and, with more prolonged fasting, also stimulation of gluconeogenesis. ${ }^{5}$

A dysregulation in the mechanisms implicated in glucose homeostasis can cause acute or chronic hyperglycemia. ${ }^{6}$ Decreased/assent insulin production and/or reduced insulin sensitivity are important contributing factors to the development of hyperglycemia and they represent the underlying abnormalities of diabetes. ${ }^{4}$ Along with a decreased/absent insulin secretion, diabetes is also characterized by impaired glucagon production, which can predispose to the risk of hypoglycemia in these patients. ${ }^{5}$ However, there is also extensive evidence that in patients with diabetes, hyperglycemia is often associated with hyperglucagonemia. ${ }^{5}$

The combined alterations in insulin and glucagon production/secretion in diabetes is the reason why recently there has been increasing interest in developing new therapeutic strategies to achieve normoglycemia based on a bihormonal approach, delivering insulin and glucagon simultaneously. ${ }^{5}$ In addition, the ongoing advances in the understanding of the complex hormonal regulation of glucose metabolism have also led to the development of new drugs to be implemented to treat hyperglycemia, such as GLP-1 or amylin analogs. ${ }^{3}$ 


\section{The spectrum of hyperglycemia}

Diabetes

Chronic hyperglycemia is the hallmark of diabetes mellitus, a chronic condition characterized not only by hyperglycemia but also by alterations in protein and lipid metabolism. ${ }^{7}$ The definition of diabetes is based on fasting glucose levels $\geq 126 \mathrm{mg} / \mathrm{dl}$ or random glucose levels $\geq 200 \mathrm{mg} / \mathrm{dl}^{7}$

Among the various forms of diabetes, type 1 diabetes (T1D) is characterized by an autoimmune destruction of pancreatic $\beta$-cells, and is the most frequent form in the pediatric population, representing more than $90 \%$ of all cases of diabetes diagnosed during childhood and adolescence. ${ }^{7}$ Over the last decades there has been a progressive increase in the incidence of T1D in children and adolescents. ${ }^{8}$ Based on recent data from the International Diabetes Federation, worldwide there are around 542,000 children younger than 14 years with T1D, with more than 86,000 newly diagnosed cases per year. ${ }^{9}$

Type 2 diabetes (T2D) is the most common form of diabetes in adults, and is characterized by the presence of a state of insulin resistance associated with a progressive loss of $\beta$-cell function. ${ }^{10}$ In recent years, concomitant with the growing epidemic of childhood obesity, there has also been the emergence of T2D among adolescents. Epidemiologic data indicate that in the US T2D now accounts for $8-87 \%$ of new cases of pediatric diabetes. ${ }^{11,12}$

Additional forms of diabetes include secondary diabetes, as a consequence of prolonged use of certain drugs, such as glucocorticoids, or occurring in the context of other diseases, such as cystic fibrosis, Cushing's syndrome; monogenic forms of diabetes, such as neonatal diabetes or the maturity onset diabetes of the young (MODY), due to defects in genes regulating insulin secretion; and gestational diabetes. ${ }^{10}$

\section{Prediabetes and other earlier forms of dysglycemia}

Prediabetes is a condition characterized by abnormal glucose concentrations, which however, are still below the cutoff for the diagnosis of diabetes. Prediabetes includes two main conditions: impaired fasting glucose (IFG), characterized by fasting glucose levels between 100 and 125 $\mathrm{mg} / \mathrm{dl}$, and impaired glucose tolerance (IGT), defined by 2-hour postload glucose levels between 140 and $199 \mathrm{mg} / \mathrm{dl}^{13,14}$ Based on epidemiologic data, about $60 \%$ of adults with T2D when assessed 5 years prior to diagnosis present either IGT or IGF.15

During more recent years there has been a lot of interest in identifying even earlier signs of dysglycemia, predictive of future risk of diabetes. Recent reports have shown that 1-hour postload glucose concentrations $\geq 155 \mathrm{mg} / \mathrm{dl}$ is a predictor of future risk of T2D in adults of different ethnic backgrounds, even in the presence of normal fasting or 2-hour postload glucose levels. ${ }^{15-19}$ In addition, this glucose cutoff is able to identify subjects with an impaired cardiometabolic profile, characterized by high blood pressure, dyslipidemia, liver steatosis, early signs of atherosclerosis, as well as an increased mortality. ${ }^{20-25}$

In children and adolescents, data on 1-hour postload glucose are not as extensive as in adults, but some preliminary studies have confirmed that a cutoff of $155 \mathrm{mg} / \mathrm{dl}$, or even of $132 \mathrm{mg} / \mathrm{dl}$, could predict future risk of T2D and identify young subjects with early cardiovascular abnormalities. ${ }^{26,27}$

\section{Complications of hyperglycemia}

High glucose concentrations can cause injury to a large number of organs and tissues. ${ }^{6}$ Most cells can adapt the rate of intracellular glucose transport under hyperglycemia conditions, and they can protect the intracellular milieu from the negative effect of high glucose levels. In contrast, other cells, such as $\beta$ cells, neuronal, and endothelial cells, are unable to activate this control of glucose afflux and they equilibrate their intracellular glucose level to the extracellular concentrations, and therefore are more susceptible to the effect of hyperglycemia. ${ }^{12,28}$

In the context of diabetes, hyperglycemia can cause acute and chronic complications, which represent important determinants of morbidity and mortality, and have a negative impact on the prognosis of people affected by this disease. ${ }^{29}$

\section{Acute complications of hyperglycemia}

Hyperglycemia can cause serious acute complications, presenting as endocrine emergencies, such as diabetic ketoacidosis (DKA) and hyperosmolar hyperglycemic state $(\mathrm{HHS})$. $^{30,31}$ Both these conditions are caused by relative or absolute insulin deficiency associated with excessive counter-regulatory hormones (glucagon, growth hormone, cortisol, catecholamines).

Although both DKA and HHS can present across the whole age spectrum, DKA tends to be more common in young people with diabetes, whereas HHS is more common among older patients..$^{30,31}$ For a long time it has been assumed that only patients with T1D were at risk of developing DKA, whereas HHS was a typical complication of patients with T2D. However, it is now clear that both conditions can occur in the context of both T1D or T2D. ${ }^{30,31} \mathrm{In}$ addition, it is also clear that there is some overlap between these two conditions. Some patients with HHS, especially when there is severe dehydration, can present mild or moderate acidosis that is mainly due to hypoperfusion/lactic acidosis. on the other hand, some patients with T1D can present some features of HHS, such as severe hyperglycemia. ${ }^{30,31}$

\section{Diabetic ketoacidosis}

DKA is an acute life-threatening complication of diabetes, characterized by the triad of hyperglycemia (>250 mg/dl), metabolic acidosis (decreased $\mathrm{pH}$ and bicarbonates), and increased total body ketone concentration. DKA represents the initial manifestation of T1D in 13-80\% of cases and it can also occur in up to $25 \%$ of cases of T2D at onset. In addition, DKA is a common complication in patients with known diabetes, where it may be the consequence of poor compliance with insulin treatment, acute illness, or malfunction of diabetes care equipment. ${ }^{30,31}$ Mortality associated with DKA is predominantly related to the occurrence of cerebral edema, which occurs in $0.3-1 \%$ of patients, whereas only a minority of deaths in DKA is due to other causes. ${ }^{32}$

Early identification and treatment of DKA are essential to minimize the associated morbidity and mortality. Treatment of DKA requires strict monitoring of the patient, correction of hyperglycemia, acidosis and ketosis, and replacement of fluid and electrolyte losses. ${ }^{30,31}$

Another important action is the identification and treatment of precipitating events. Prevention of DKA at diagnosis is of paramount importance and should be based on intensive community interventions and education of 
healthcare providers to raise awareness. In addition, preventive strategies should be applied to avoid episodes of DKA in patients with an already known diagnosis of diabetes. This requires patient education and access to specific diabetes programs and services.

\section{Hyperosmolar hyperglycemic state}

HHS is the most serious acute hyperglycemic emergency in patients with T2D. Diagnostic criteria for this condition are glucose level $>600$ $\mathrm{mg} / \mathrm{dl}$ and increased effective plasma osmolality $>320 \mathrm{mosm} / \mathrm{kg}$, in the absence of ketoacidosis. ${ }^{30,31}$ The incidence of HHS is estimated to be $<1 \%$ of hospital admissions of patients with diabetes and the associated mortality is $10-20 \%$. There are no good data from randomized studies on the best management of HHS, which has been mainly extrapolated from studies of patients with DKA. In HHS, the goals of initial fluid therapy are to expand the intra- and extravascular volume, restore normal renal perfusion, and promote a gradual decline in serum sodium concentration and osmolality. ${ }^{30,31}$

\section{Chronic complications of diabetes-vascular complications}

Hyperglycemia is a key determinant of vascular complications of diabetes, also known as chronic diabetes complications. ${ }^{33,34}$ There is extensive evidence indicating that both acute and chronic hyperglycemia play a key role in the pathogenesis of these complications (see Figure 1).

Vascular complications of diabetes are divided into "microvascular" (such as retinopathy, nephropathy, and neuropathy) resulting from damage of the small vessels within the microcirculation of the kidney, retina, and neurons and "macrovascular," reflecting damage of large vessels and leading to cardiovascular disease. ${ }^{34}$

As a result of microvascular complications, diabetes is an important determinant of blindness, end-stage renal disease, and a variety of debilitating neuropathies. Diabetic nephropathy is a common microvascular complication of diabetes, which manifests with progressive increases in urinary albumin excretion, along with changes in glomerular filtration rate, ultimately leading to the development of end-stage renal disease. Diabetic nephropathy represents the major cause of end-stage renal disease in both developed and developing countries and it is an independent risk factor for cardiovascular disease. ${ }^{35}$

Diabetic retinopathy is one of the leading causes of blindness in people of working age. This complication can be diagnosed already after five years from the onset of diabetes, and almost all patients will show variable degrees of retinopathy after 20 years of diabetes. ${ }^{36}$

Diabetic peripheral neuropathy is a common complication estimated to affect $30-50 \%$ of individuals with diabetes, although clinical symptoms do not generally occur until long after the onset of diabetes. Abnormalities of the autonomic nervous system can also occur in patients with diabetes, with early subclinical manifestations, such as decreased heart rate variability, being detectable within a year of diagnosis in patients with T2D, and within two years in patients with T1D. ${ }^{37}$

With regard to macrovascular complications, epidemiologic data indicate that people with diabetes have a two- to fourfold increased risk of developing cardiovascular disease, which in turn is a key contributor of mortality.38
Figure 1: Different components of hyperglycemia in relation to complication risk

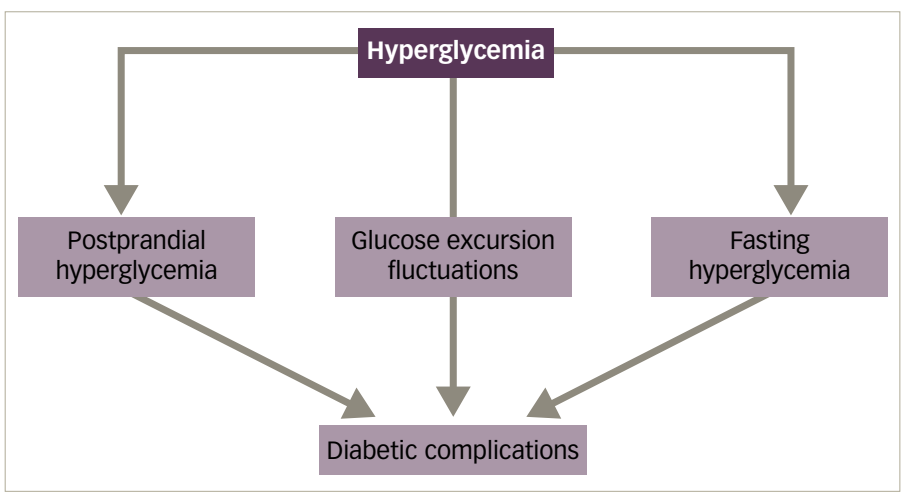

Hyperglycemia and vascular damage

Hyperglycemia contributes to the development of vascular complications through several mechanisms: activation of the polyol and hexosamine pathways, diacylglycerol-protein kinase C (DAG-PKC), increased production of advanced glycation end products (AGE), increased synthesis of growth factors, cytokines, and oxidative stress. ${ }^{28,39}$ The polyol pathway is implicated in the pathogenesis of vascular complications through the action of aldose reductase, the first and rate-limiting enzyme in this pathway. Aldose reductase reduces the aldehyde form of glucose to sorbitol, which is then oxidized to fructose by sorbitol dehydrogenase and then again enters into glycolysis. In the context of hyperglycemia, the production of sorbitol overcomes the potential of its oxidation, with its consequent accumulation within the cells and dysregulation of the cellular osmotic status, reduction of $\mathrm{Na}^{+} / \mathrm{K}^{+}-\mathrm{ATPase}$ activity, cytosolic increase in $\mathrm{NADH} /$ $\mathrm{NAD}^{+}$, and decrease in NADPH. ${ }^{28}$

The hexosamine pathway converts fructose-6-phosphate into $\mathrm{N}$-acetyl glucosamine, which has been implicated in the activation of the transcriptional factor Sp1, leading to increased synthesis of factors, such as transforming growth factor beta-1 (TGF-B1) and plasminogen activator inhibitor-1 (PAl-1), which in turn are associated to the development of vascular complications. In addition, the hexosamine pathway is also associated with increased oxidative stress and the effects of the activation of this pathway can be prevented by overexpression of antioxidants, such as superoxide dismutase. Hyperglycemia can also stimulate de novo synthesis of DAG, followed by the activation of PKC, which in turn modulates the activity of various enzymes, including phospholipase A2, $\mathrm{Na}^{+} / \mathrm{K}^{+}-$ATPase, as well as the expression of genes related to components of the extracellular matrix. ${ }^{28}$

AGEs have been implicated in several biologic activities, mostly by binding to the AGE-specific receptors (RAGES) on many cells. In particular, they can induce oxidative stress and release of cytokines and growth factors, which in turn accelerate chronic inflammation and endothelial dysfunction. ${ }^{28}$

Growing evidence suggests that increased oxidative stress, induced by the above hyperglycemia-activated pathways, is a key factor in the pathogenesis of endothelial dysfunction and vascular disease. Several mitochondrial and other intracellular pathways are implicated in the increased production of oxidant species concomitant with a reduction in antioxidants in the context of diabetes. ${ }^{28,39}$ 
In addition to the above pathways/mechanisms, recent studies have also suggested the involvement of hyperglycemia-induced epigenetic changes and microRNA levels in the pathogenesis of diabetes vascular complications. ${ }^{40}$

\section{Intervention to improve glycemic control}

The Diabetes Control and Complications Trial (DCCT) and its follow-up study, the Epidemiology of Diabetes Interventions and Complications (EDIC), have clearly shown the role of strict glycemic control in reducing the risk of vascular complications in subjects with T1D. ${ }^{40-42}$

The DCCT studied a cohort of 1,441 subjects, aged 13-39 years, with T1D for 1-15 years ${ }^{42}$ by comparing intensive (insulin administered three or more times daily by injection or an external pump) versus conventional (one or two daily insulin injections) insulin therapy. Intensive insulin therapy reduced the risk for the development of retinopathy by $76 \%$, slowed retinopathy progression by $54 \%$, and reduced the development of proliferative or severe nonproliferative retinopathy by $47 \%$. Intensive insulin therapy reduced the occurrence of diabetic nephropathy by $39-54 \%$ and that of clinical neuropathy by $60 \%$. In the DCCT intensive treatment also reduced the risk of any cardiovascular disease event by $42 \%$ and the risk of nonfatal myocardial infarction, stroke, or death from cardiovascular disease by $57 \% .^{42}$ Although already after 2 years from the end of the DCCT, HDA1C levels were similar between the previously intensively and conventionally treated groups, complication rates in the previously intensive treated group were still lower, thus postulating the concept of the "metabolic memory." ${ }^{43}$ That is, patients who were previously exposed to better glycemic levels continued to have an advantage in terms of protection from the development of chronic complications several years later. Therefore, the EDIC study highlighted the need of implementing intensive management as soon as diabetes is diagnosed. This was further confirmed by more recent EDIC data showing significant differences in the persistence of the "metabolic memory" between the DCCT adolescent and adult cohorts. ${ }^{44}$ At year 10 of EDIC the advantage of the previous intensively insulin treatment in terms of diabetic retinopathy progression still persisted in the adult cohort, whereas it did not in the adolescent cohort. These contrasting findings between adults and adolescents were largely explained by the difference in $\mathrm{HbA} 1 \mathrm{C}$ during the DCCT years, when, within the intensively treated group, adolescents consistently showed a mean $\mathrm{HbA} 1 \mathrm{C} 1 \%$ higher than adults. ${ }^{44}$

The mechanisms underpinning this "metabolic memory" remain unclear However, recent data suggest that epigenetic modulations, such as histones and DNA methylation, may be involved in persistent changes of gene expression associated with vascular complications of diabetes and lead to metabolic memory. ${ }^{45,46}$ Of interest, a recent study demonstrated that specific microRNA (i.e., miR-125b and miR-146a-5) changes can cause persistent increase in proatherogenic gene expression and explain in this way the phenomenon of metabolic memory. ${ }^{47}$

The key role of glycemic control in modulating complication risks highlighted by the DCCT/EDIC studies was also confirmed by large studies performed in adults with T2D, such as the UKPDS, where for example intensive treatment (insulin or sulfonylurea) resulted in a $25 \%$ decrease in microvascular complications. ${ }^{48}$

\section{Chronic versus acute hyperglycemia in the pathogenesis of vascular complications}

Hemoglobin A1C ( $\mathrm{HbA1C}$ ) is the main parameter which has been used for over 30 years to monitor long-term glycemic control. ${ }^{49} \mathrm{HbA} 1 \mathrm{C}$ levels reflect blood glucose levels over a 2- to 3-month period, with plasma glucose levels in the preceding 30 days contributing $50 \%$ to the final result, and plasma glucose levels from $90-120$ days earlier contributing only $10 \%{ }^{50}$ The DCCT/EDIC, UKPDS, and several other studies have clearly shown the strong association between $\mathrm{HbA} 1 \mathrm{C}$ and vascular complications of

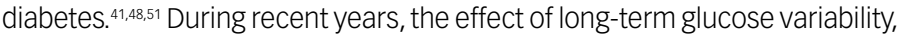
as assessed by the intraindividual variability of HbA1C values across visits, on retinopathy, nephropathy, and cardiovascular complications has also been documented..$^{52}$

However, HbA1c measurement does not give any information about individual daily glucose fluctuations (short-term fluctuations or acute hyperglycemia). In patients with marked fluctuations in glucose concentrations who are exposed to the risk of both hyperglycemia and hypoglycemia, HbA1c levels may still indicate adequate metabolic control. ${ }^{53}$

Extensive evidence indicates that short-term fluctuations in glucose (acute hyperglycemia) can play a key role in the pathogenesis of diabetic vascular complications, independently from the effect on HbA1C. ${ }^{53}$ Acute hyperglycemia can be due to high FPG and/or high PPG levels. The contribution of FPG and PPG to long-term glycemic control varies across the range of $\mathrm{HbA} 1 \mathrm{c}$ concentrations. In particular, it has been shown that, whereas the relative contribution of PPG decreases from the lowest to the highest quintiles of $\mathrm{HbA} 1 \mathrm{C}$, the relative contribution of FPG increases with higher levels of HbA1C.53

Acute hyperglycemia has been associated with increased renal perfusion, hyperfiltration, increased oxidative stress, decreased motor and sensory nerve conduction, increased collagen production in the kidney, and increased retinal perfusion. All these mechanisms can contribute to the development of microvascular complications. ${ }^{53}$ In addition, short glucose excursions can induce endothelial dysfunction, increase oxidative stress, activate coagulation factors, increase the expression of adhesion molecules, increase blood pressure, and dyslipidemia. Again, all these mechanisms can contribute to the development of macrovascular complications. ${ }^{53}$

Although there is some increasing evidence for a role of acute hyperglycemia, such as PPG, in the development of vascular complications, there is still a need of further data, mainly obtained from interventional studies exploring drugs specifically targeting PPG.

Short-term glucose variability, which represents the intraday glucose fluctuations from peaks to nadirs, is another parameter, reflecting short-glucose fluctuations and which has been investigated in relation to diabetic vascular complications. ${ }^{54}$

In vitro studies have shown a significant effect of glucose fluctuations in the activation of oxidative stress pathways, induction of epigenetic changes in key genes, and endothelial dysfunction. Several studies performed in patients with T2D have shown a direct association between glucose variability and the development or progression of retinopathy, cardiovascular disease and mortality.5,56 in contrast, some studies in patients with T1D have shown that glucose variability has a small effect on the development of diabetic complications, with few studies reporting an association with microvascular complications, whereas it appears that there is no link with macrovascular disease. ${ }^{55,56}$ Data from the DCCT showed no association between measures of glucose variability and microvascular 
complications. ${ }^{57}$ Similarly, another study in patients with T1D could not confirm the association found in patients with T2D between oxidative stress and glucose variability. ${ }^{55}$ Further studies are required to clarify the role of glucose variability as a potential additional component in the network of vascular complications of diabetes.

\section{Conclusions}

Hyperglycemia is a well-known metabolic derangement, which can contribute to the development of serious acute (DKA, HHS) and chronic complications (micro- and macrovascular disease). Several studies have clearly shown a strong association between chronic hyperglycemia and the development of both micro- and macrovascular complications of diabetes. In addition, there is growing evidence suggesting that acute hyperglycemia, particularly PPG, plays a role in the pathogenesis of complications. In contrast, the role of glucose variability in the development of vascular complications is not yet clear.

Interventions able to reduce $\mathrm{HbA} 1 \mathrm{C}$ can reduce the development or progression of vascular complications of diabetes. Further studies are required to clarify the impact of strategies targeting more specifically components of acute hyperglycemia, to improve outcomes in patients with diabetes. $\square$
1. Shrayyef MZ, Gerich JE, Normal Glucose Homeostasis. In Poretsky $\mathrm{L}$ (ed), Principles of Diabetes Mellitus, Boston, MA: Springer US, 2010;19-35

2. Szablewski L, Glucose Homeostasis and Insulin Resistance, Bentham Science Publishers; 2011. doi: 10.2174/97816080518921110101

3. Aronoff SL, Berkowitz K, Shreiner B, Want L, Glucose metabolism and regulation: beyond insulin and glucagon, Diabetes Spectr. 2004:17:183-90.

4. Rizza RA, Mandarino LJ, Gerich JE, Dose-response characteristics for effects of insulin on production and utilization of glucose in man, Am J Physiol, 1981;240:E630-9.

5. Campbell JE, Drucker DJ, Islet $\alpha$ cells and glucagon — critical regulators of energy homeostasis, Nat Rev Endocrino 2015:11:329-38.

6. Giugliano D, Ceriello A, Esposito K, Glucose metabolism and hyperglycemia, Am J Clin Nutr, 2008;87:217S-22S.

7. American Diabetes Association, 2. Classification and diagnosis of diabetes, Diabetes Care, 2017;40:S11-24.

8. Patterson $C C$, Dahlquist $G G$, Gyürüs $E$, et al., Incidence trends for childhood type 1 diabetes in Europe during 1989-2003 and predicted new cases 2005-20: a multicentre prospective registration study, Lancet, 2009;373:2027-33.

9. IDF diabetes atlas - Home [Internet], 2017. Available at: www.diabetesatlas.org/ (accessed February 26, 2017)

10. American Diabetes Association, 2. Classification and diagnosis of diabetes, Diabetes Care, 2017;40:S11-24.

11. D'Adamo E, Caprio S, Type 2 diabetes in youth: epidemiology and pathophysiology, Diabetes Care, 2011;34:S161-5.

12. Nadeau KJ, Anderson BJ, Berg EG, et al., Youth-onset type 2 diabetes consensus report: current status, challenges, and priorities, Diabetes Care, 2016:39:1635-42.

13. Unwin $\mathrm{N}$, Shaw J, Zimmet P, Alberti KG, Impaired glucose tolerance and impaired fasting glycaemia: the current status on definition and intervention, Diabet Med, 2002;19:708-23.

14. Abdul-Ghani MA, Lyssenko V, Tuomi T, et al., The shape of plasma glucose concentration curve during OGTT predicts future risk of type 2 diabetes, Diabetes Metab Res Rev, 2010;26:280-6.

15. Abdul-Ghani MA, Williams $K$, DeFronzo RA, Stern $M$, What is the best predictor of future type 2 diabetes? Diabetes Care, 2007:30:1544-8.

16. Abdul-Ghani MA, Abdul-Ghani T, Ali N, Defronzo RA, One-hour plasma glucose concentration and the metabolic syndrome identify subjects at high risk for future type 2 diabetes, Diabetes Care, 2008;31:1650-5.

17. Abdul-Ghani MA, Lyssenko V, Tuomi T, et al., Fasting versus postload plasma glucose concentration and the risk for future type 2 diabetes: results from the Botnia study, Diabetes Care 2009;32:281-6.

18. Alyass $A$, Almgren $P$, Akerlund $M$, et al., Modelling of OGTT curve identifies $1 \mathrm{~h}$ plasma glucose level as a strong predictor of incident type 2 diabetes: results from two prospective cohorts, Diabetologia, 2014;58:87-97.

19. Jagannathan R, Sevick MA, Li H, et al., Elevated 1-hour plasma glucose levels are associated with dysglycemia, impaired beta-cell function, and insulin sensitivity: a pilot study from a rea world health care setting, Endocrine, 2015;52:172-5.

20. Succurro E, Marini MA, Arturi F, et al., Elevated one-hour post-load plasma glucose levels identifies subjects with normal glucose tolerance but early carotid atherosclerosis, Atherosclerosis, 2009;207:245-9.
21. Bianchi $C$, Miccoli $R$, Trombetta M, et al., Elevated 1-hour postload plasma glucose levels identify subjects with normal glucose tolerance but impaired $\beta$-cell function, insulin resistance, and worse cardiovascular risk profile: the GENFIEV study, J Clin Endocrinol Metab, 2013;98:2100-5.

22. Sciacqua A, Miceli S, Carullo G, et al., One-hour postload plasma glucose levels and left ventricular mass in hypertensive patients, Diabetes Care, 2011:34:1406-11.

23. Sesti G, Hribal ML, Fiorentino TV, et al., Elevated $1 \mathrm{~h}$ postload plasma glucose levels identify adults with normal glucose tolerance but increased risk of non-alcoholic fatty liver disease, BMJ Open Diabetes Res Care, 2014;2:e000016.

24. Orencia AJ, Daviglus ML, Dyer AR, et al., One-hour postload plasma glucose and risks of fatal coronary heart disease and stroke among nondiabetic men and women: the Chicago Heart Association Detection Project in Industry (CHA) study, J Clin Association Detection Project

25. Bergman M, Chetrit A, Roth J, et al., One-hour post-load plasma glucose level during the OGTT predicts dysglycemia, Diabetes Res Clin Pract, 2016;120:221-8.

26. Manco M, Miraglia Del Giudice E, Spreghini MR, et al., 1-Hour plasma glucose in obese youth, Acta Diabetol, 2012;49:435-43.

27. Tfayli $\mathrm{H}$, Jung Lee $\mathrm{S}$, Bacha F, Arslanian $\mathrm{S}$, One-hour plasma glucose concentration during the OGTT: what does it tell about $\beta$-cell function relative to insulin sensitivity in overweight/obese children?, Pediatr Diabetes, 2011;12:572-9.

28. Brownlee $\mathrm{M}$, The pathobiology of diabetic complications: a unifying mechanism, Diabetes, 2005;54:1615-25.

29. Orasanu G, Plutzky J, The pathologic continuum of diabetic vascular disease, J Am Coll Cardiol, 2009:53:S35-42.

30. Umpierrez G, Korytkowski M, Diabetic emergencies - ketoacidosis, hyperglycaemic hyperosmolar state and hypoglycaemia, Nat Rev Endocrinol, 2016;12:222-32.

31. Steenkamp DW, Alexanian SM, MCDonnell ME, Adult hyperglycemic crisis: a review and perspective, Curr Diab Rep, 2013;13:130-7

32. Wolfsdorf Jl, Allgrove J, Craig ME, et al., Diabetic ketoacidosis and hyperglycemic hyperosmolar state, Pediatr Diabetes, 2014:15:154-79.

33. Marcovecchio ML, Tossavainen PH, Dunger DB, Prevention and treatment of microvascular disease in childhood type 1 diabetes, Br Med Bull, 2010;94:145-64.

34. Orasanu G, Plutzky J, The pathologic continuum of diabetic vascular disease, J Am Coll Cardiol, 2009;53:S35-42.

35. Gross IL, de Azevedo MJ, Silveiro SP, et al., Diabetic nephropathy: diagnosis, prevention, and treatment, Diabetes Care, 2005;28:164-76.

36. Solomon SD, Chew E, Duh EJ, et al., Diabetic retinopathy: a position statement by the American Diabetes Association, Diabetes Care 2017:40:412-8.

37. Pop-Busui R, Boulton AJM, Feldman EL, et al., Diabetic neuropathy: a position statement by the American Diabetes Association, Diabetes Care, 2017;40:136-54.

38. Duca L, Sippl R, Snell-Bergeon JK, Is the risk and nature of CVD the same in Type 1 and Type 2 diabetes?, Curr Diab Rep, 2013;13:350-61.

39. Forbes JM, Cooper ME, Mechanisms of diabetic complications, Physiol Rev, 2013;93:137-88

40. Pirola L, Nathan D, Zinman B, et al., The DCCT/EDIC study: epigenetic clues after three decades, Diabetes, 2014;63:1460-2.
41. Nathan DM, MCGee P, Steffes MW, Lachin JM, DCCT/EDIC Research Group, Relationship of glycated albumin to blood glucose and $\mathrm{HDA} 1 \mathrm{C}$ values and to retinopathy, nephropathy, and cardiovascular outcomes in the DCCT/EDIC study, Diabetes, 2014;63:282-90.

42. Group TDC and CTR, The effect of intensive treatment of diabetes on the development and progression of long-term complications in insulin-dependent diabetes mellitus, N Engl J Med, 1993;329:977-86.

43. Kilpatrick ES, Rigby AS, Atkin SL, The diabetes control and complications trial: the gift that keeps giving, Nat Rev Endocrinol, 2009;5:537-45.

44. White NH, Sun W, Cleary PA, et al., Effect of prior intensive therapy in type 1 diabetes on 10-year progression of retinopathy in the DCCT/EDIC: comparison of adults and adolescents, Diabetes, 2010;59:1244-53.

45. Pirola L, The DCCT/EDIC study: epigenetic clues after three decades, Diabetes, 2014;63:1460-2.

46. Chen Z, Miao F, Paterson AD, et al., Epigenomic profiling reveals an association between persistence of DNA methylation and metabolic memory in the DCCT/EDIC type 1 diabetes cohort, Proc Natl Acad SCi, 2016;201603712

47. Zhong $X$, Liao $Y$, Chen $L$, et al., The microRNAs in the pathogenesis of metabolic memory, Endocrinology, 2015;156:3157-68.

48. Intensive blood-glucose control with sulphonylureas or insulin compared with conventional treatment and risk of complications in patients with type 2 diabetes (UKPDS 33). UK Prospective Diabetes Study (UKPDS) Group, Lancet, 1998;352:837-53.

49. Nathan DM, Singer DE, Hurxthal K, Goodson JD, The clinica information value of the glycosylated hemoglobin assay, N Eng/ J Med, 1984;310:341-6.

50. Tahara Y, Shima K, The response of GHb to stepwise plasma glucose change over time in diabetic patients, Diabetes Care 1993;16:1313-4

51. Marcovecchio ML, Dalton RN, Chiarelli F, Dunger DB, A1C variability as an independent risk factor for microalbuminuria in young people with type 1 diabetes, Diabetes Care, 2011:34:1011-3.

52. Gorst C, Kwok CS, Aslam S, et al., Long-term glycemic variability and risk of adverse outcomes: a systematic review and meta-analysis, Diabetes Care, 2015;38:2354-69.

53. Monnier L, Lapinski $\mathrm{H}$, Colette $\mathrm{C}$, Contributions of fasting and postprandial plasma glucose increments to the overall diurnal hyperglycemia of type 2 diabetic patients: variations with increasing levels of $\mathrm{HbA}(1 \mathrm{C})$, Diabetes Care, 2003;26:881-5.

54. Suh S, Kim JH, Glycemic variability: how do we measure it and why is it important? Diabetes Metab J, 2015;39:273.

55. Nalysnyk L, Hernandez-Medina M, Krishnarajah G, Glycaemic variability and complications in patients with diabetes mellitus: evidence from a systematic review of the literature, Diabetes Obes Metab, 2010;12:288-98.

56. Smith-Palmer J, Brändle M, Trevisan R, et al., Assessment of the association between glycemic variability and diabetes-related complications in type 1 and type 2 diabetes, Diabetes Res Clin Pract, 2014:105:273-84.

57. Kilpatrick ES, Rigby AS, Atkin SL, The effect of glucose variability on the risk of microvascular complications in Type 1 diabetes, Diabetes Care, 2006;29:1486-90. 